\title{
The Efficacy of Standardized Electronic Insulin Orders In Controlling Blood Glucose Levels In Internal Medicine Departments
}

\author{
Dicker $D^{1,2 *}$, Marcoviciu $D^{1}$, Beylin $V^{1}$, Belnik $Y^{1}$ and Solun $B^{1}$
}

${ }^{1}$ Department of Internal Medicine D, Rabin Medical Center, Hasharon Hospital, Petah Tikva, Israel

${ }^{2}$ Sackler School of Medicine, Tel-Aviv University, Ramat Aviv, Israel

\begin{abstract}
Background: Hyperglycemia in hospitalized patients is found to be associated with increased risk of complications, systemic infections and mortality. Thus, managing and controlling glucose blood levels in these patients are essential. The aim of the present retrospective study was to evaluate the efficacy of standardized electronic insulin orders, using a computerized physician order entry (CPOE), in controlling blood glucose levels, preventing hypoglycemic events and reducing the variability of daily blood glucose levels during hospitalization of patients with diabetes mellitus in internal medicine departments.

Methods: Data were obtained from an internal medicine unit that used the standardized electronic insulin orders (Department 7) and compared to data from 6 other internal medicine departments in which the standardized electronic insulin orders was not used (Departments 1-6). The variability of daily changes in blood glucose levels was determined by the standard deviation of the daily changes and respective coefficient of variation.

Results: 130,078 point-of-care bedside glucose tests were recorded from 13,577 hyperglycemic patients. The average daily mean blood glucose levels were significantly lower in patients from Department 7 than from Departments 1-6. The rate of patients achieving average daily mean blood glucose levels $\leq 180 \mathrm{mg} / \mathrm{dL}$ was higher in Department 7 than Departments 1-6. The percent of patient days with hypoglycemia and the variability of daily changes in blood glucose levels were significantly lower in Department 7 than Departments 1-6.
\end{abstract}

Conclusions: A standardized electronic insulin orders of basal/bolus regimen improves glucose control in hospitalized patients.

\section{Background}

The prevalence of diabetes mellitus (DM), both type 1 and 2 , is a growing public health burden [1]. According to the 2011 report of the Centers for Disease Control and Prevention (CDC), 25.8 million people of all ages in the U.S ( $8.3 \%$ of all the USA population) were diagnosed with DM. The total estimated cost of DM treatment was $\$ 245$ billion in 2012 (an increase in cost of $41 \%$ since 2007) [2].

Patients with DM are much more likely to be hospitalized and to experience a longer hospitalization time than those without a known history of DM. It is estimated that about $22 \%$ of the total days of hospitalization are related to patients with DM. Based on the CDC report of 2010, DM was the primary discharge diagnosis for $11.5 \%$ of hospitalized patients [2].

Hyperglycemia in hospitalized patients (with or without diabetes) was found to be associated with increased risk of complications, systemic infections and mortality [3-5, CDC Diabetes-Data and Trends-Hospitalization, 2010]. In patients who underwent surgery, the risk of developing non-fatal stroke, septic complication or death after 24 hours, increased 2.7 -fold when postoperative blood glucose exceeded $225 \mathrm{mg} / \mathrm{dl}$ [6]. Krinsley et al. reported mortality rates of $9.6 \%$ and $42.5 \%$ among intensive care patients with normal and high (exceeding $300 \mathrm{mg} / \mathrm{dL}$ ) glucose concentrations, respectively [7]. Moreover, the mortality rate was found to rise with every $10 \mathrm{mg} / \mathrm{dl}$ increase in glucose above $120 \mathrm{mg} / \mathrm{dl}$, in patients hospitalized with acute myocardial infarction, especially in those without a history of DM [8]. In light of these observations, managing and controlling glucose blood levels in hospitalized patients are essential to reduce morbidity, mortality and health care costs. Insulin is the most effective agent for immediate control of hyperglycemia in the hospital.
A variety of insulin therapy protocols have been shown to be effective in achieving glycemic control, with a low rate of hypoglycemic events, consequently improving hospitalization outcomes. A basal-bolus insulin regimen combines a long-lasting insulin analog to prevent hyperglycemia upon fasting and between-meals; together with a number of bolus dosages of rapid-acting analog throughout the day, to prevent hyperglycemia after food intake. The 2015 American Diabetes Association Standards of Medical Care in Diabetes has recommended a multidisciplinary approach to glucose management, which incorporates standardized electronic insulin orders in hospital protocols [9].

The aim of the present study was to compare blood glucose levels, hypoglycemic events and variability of daily blood glucose levels, between patients with diabetes hospitalized in one internal medicine department that uses standardized electronic insulin orders of basalbolus regimen and between patients hospitalized in internal medicine departments that do not use such a system.

*Corresponding author: Dr. Dror Dicker, Rabin Medical Center - Hasharon Hospital, 7 Keren Kayemet St., Petah Tiqva, 49100-Israel, Tel: 972-3- 9372426 Fax: 972-3-9372427; E-mail: daniel3@013.net

Received March 13, 2015; Accepted April 20, 2015; Published April 24, 2015

Citation: Dicker D, Marcoviciu D, Beylin V, Belnik Y, Solun B (2015) The Efficacy of Standardized Electronic Insulin Orders In Controlling Blood Glucose Levels In Internal Medicine Departments. J Diabetes Metab 6: 538. doi:10.4172/21556156.1000538

Copyright: (C) 2015 Dicker D, et al. This is an open-access article distributed under the terms of the Creative Commons Attribution License, which permits unrestricted use, distribution, and reproduction in any medium, provided the original author and source are credited. 


\section{Methods}

For this retrospective study, all point-of-care bedside glucose (POC-BG) tests performed in diabetic patients hospitalized in 7 internal medicine wards between August 2010 and December 2012 were accessed. At our medical facility, nonsurgical patients are arbitrarily hospitalized in one of the 7 internal medicine departments. Patients who undergo acute coronary events and who are candidates for percutaneous coronary intervention are hospitalized in intensive coronary care units, and not internal medicine units. During the study period, the most common diagnoses in the 7 departments that were included in the current study were: chest pain, pulmonary disorders, and infections. Patients who did not survive hospitalization were not included in the analysis.

Data were compared between one internal medicine department (Department 7), which utilized standardized electronic insulin orders of basal/bolus regimen ["Chameleon" EMR, Computerized Physician Order Entry (CPOE)], and between 6 other internal medicine departments in which the standardized electronic insulin orders were not used (Departments 1-6). The Chameleon CPOE is an electronic medical record information system that stores and provides patient administrative and clinical information to hospital staff (http://www. elad-solutions.com).

In Department 7, a multidisciplinary team including physicians, nurses trained in DM management and a dietitian collaborated in their utilization of the CPOE to achieve desired blood glucose values for hospitalized patients. The therapeutic approach was based on a basalbolus protocol that was implemented for all patients with an average of 2 POC-BG values exceeding $200 \mathrm{mg} / \mathrm{dl}$ at admission. Regular POC-BG measurements were collected 3-4 times daily, before meals and at bedtime. The CPOE displayed blood glucose curves and physician orders for basal, bolus and correction doses of insulin. Types of insulin used were: the long acting glargine (Lantus, Sanofi, Paris France) and detemir (Levemir, Novo Nordisk Inc. Plainsboro, NJ USA) and the short acting NovoRapid (Novo Nordisk), lispro (Humalog, Eli Lilly, Indianapolis, Indiana, USA) and glulisine (Apidra, Sanofi-Aventis). The total daily dose (TDD) of insulin was calculated according to $0.3-0.5$ unit $/ \mathrm{kg}$. Physicians entered the appropriated TDD calculation $(0.3,0.4$ or $0.5 \mathrm{unit} / \mathrm{kg})$ and the CPOE displayed the daily basal and bolus doses; with $50 \%$ of the TDD given as basal and $50 \%$ as bolus insulin. The total bolus insulin was divided into 3 doses, and administered before meals. When POC-BG was $>180 \mathrm{mg} /$ $\mathrm{dL}$ before a meal, the CPOE displayed the correction dose of short acting insulin to be added, by the attending nurse, to the calculated pre-meal short acting insulin (glucose blood levels $180-250 \mathrm{mg} / \mathrm{dL}$ - addition of 2 units, $180-250 \mathrm{mg} / \mathrm{dL}$ - addition of 3 units, $300-350 \mathrm{mg} / \mathrm{dL}$ - addition of 4 units, and when glucose blood levels were $>350 \mathrm{mg} / \mathrm{dL}$ a physician was consulted). In the case of a hypoglycemic event, TDD was reduced by $20 \%$. A flowchart of the treatment protocol was implemented in the CPOE "Chameleon" EMR system.

Statistical analyses were performed using SAS $^{\circ}$ V9.3 $\left(\right.$ SAS $^{\circ}$ Institute, Cary NC, USA). Continuous data were summarized by means and standard deviations, and categorical data by a count and percentage. Categorical variables were compared with a chi-squared test. Daily mean blood glucose levels were compared using repeated measures analysis of variance (RMA) (SAS ${ }^{\circledR}$ PROC MIXED), lsmeans (model estimated means) were presented with standard errors. Pairwise differences between the lsmeans were compared between the departments. Using a repeated measures mixed model we also calculated the variability of the daily changes in glucose levels per department. The model estimated mean glucose value was calculated from the model intercept and the overall daily variability, i.e. the standard deviation of the daily fluctuations in BG levels was calculated from the model variance components, and is presented with $95 \%$ confidence intervals. The coefficient of variation, which is a measure of relative variability comparable across studies, is also presented as a percentage $\left(\mathrm{CV} \%=100^{*}[\mathrm{mean} / \mathrm{SD}]\right)$. The proportion of hypoglycemic patient days was calculated by checking if the daily mean value was $<70$ $\mathrm{mg} / \mathrm{dL}$ and calculating the ratio: number of patient days with mean $<70$ $\mathrm{mg} / \mathrm{dL} /$ number patient days.

\section{Results}

During the study period, 130,078 POC-BGs were collected

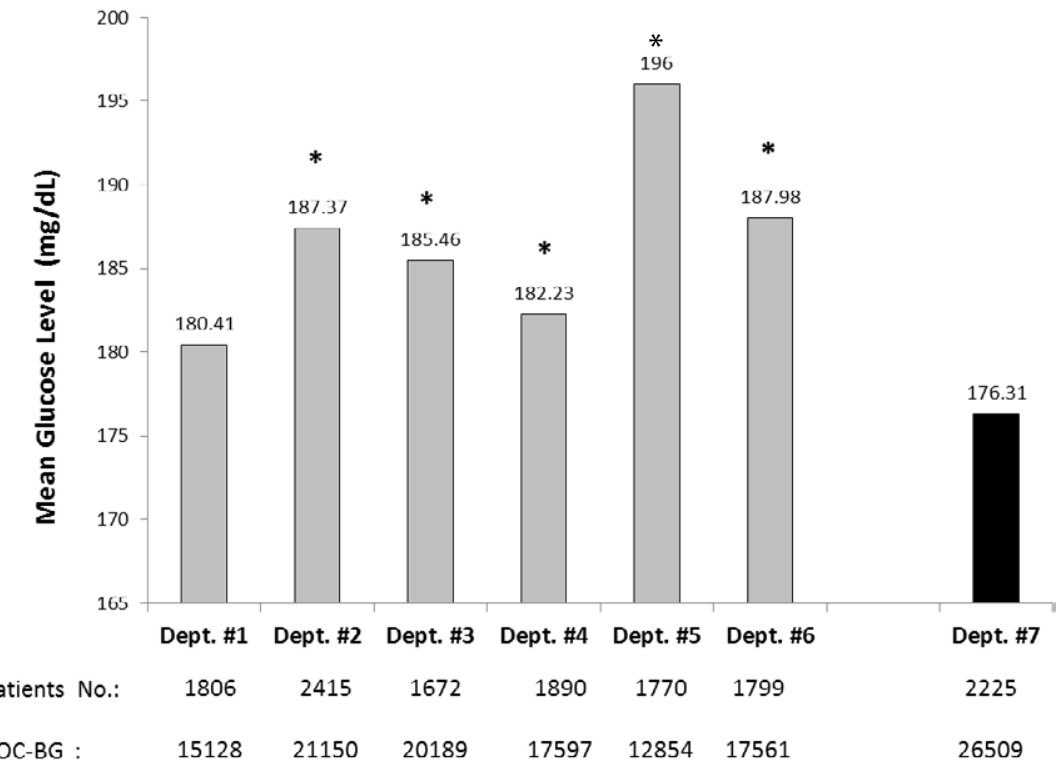

Figure 1: Mean daily blood glucose values by department, number of patients and POC-BG. ${ }^{*}-p<0.001$ 
from 13,577 hyperglycemic patients who were hospitalized either in Department 7, which utilized a standardized electronic insulin orders of basal/bolus regimen, or in Departments 1-6, which did not use a standardized electronic insulin orders.

The average daily mean blood glucose levels were significantly lower in patients who were hospitalized in Department 7 than in patients in Departments 1-6 $(176.3 \pm 69.51 \mathrm{mg} / \mathrm{dL}$ versus $186.4 \pm 38$ $\mathrm{mg} / \mathrm{dL}$ respectively, $\mathrm{p}<0.001$ ) (Figure 1 ). Moreover, the rate of patients achieving average daily mean blood glucose levels $\leq 180 \mathrm{mg} / \mathrm{dL}$ was higher in those who were hospitalized in Department 7 than in those hospitalized in Departments 1-6 (62.4\% vs. $57.6 \%, \mathrm{p}<0.001)$ (Figure 2 ). In Department 7 , the percent of hypoglycemic patients days, expressed as the ratio of the number of patient days with a mean < $70 \mathrm{mg} / \mathrm{dL} /$ number of patient days, was $0.25 \%$, compared to $0.48 \%$ in Departments 1-6 ( $\mathrm{p}=0.0014)$ (Figure 3$)$. The variability of daily changes in blood glucose levels was measured by calculation of the standard deviation of the daily changes and the coefficient of variation (CV\%). The mean variability of the daily changes in glucose blood levels was significantly less for patients hospitalized in Department 7 than for those hospitalized in Departments 1-6 (Table 1): $88.16 \mathrm{mg} / \mathrm{dl}$ (95\%CI:[87.307 - 89.032]) vs. $99.16 \mathrm{mg} / \mathrm{dl}$ (95\% CI: [98.699 - 99.624]). The coefficients of variation were $48.9 \%$ and $51.9 \%$ respectively. There was no difference among the departments in mean length of hospital stay.
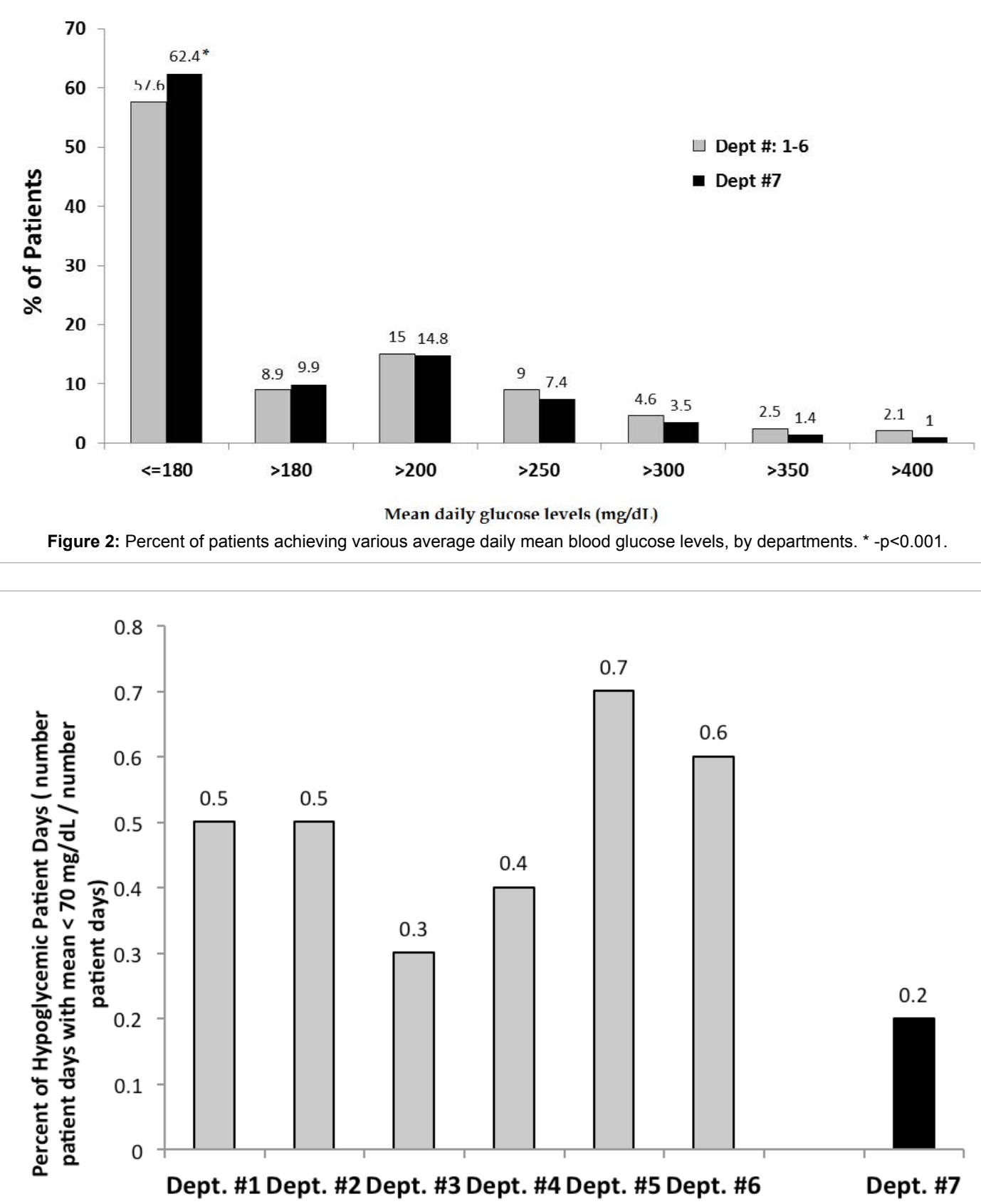

Figure 3: Percent of hypoglycemic patient days, by department. * $p=0.0014$, for Department 7 compared to Departments $1-6$. 


\begin{tabular}{|c|c|c|c|c|c|}
\hline Department & Mean & $95 \% \mathrm{Cl}$ & Within Day Variability (SD) & $95 \% \mathrm{Cl}$ & CV\% \\
\hline Dept. \#1 & 186.13 & [184.19-188.07] & 99.007 & [97.804-100.24] & $53.2 \%$ \\
\hline Dept. \#2 & 192.91 & [191.29-194.53] & 98.495 & [97.486-99.526] & $51.1 \%$ \\
\hline Dept. \#3 & 189.18 & [187.47-190.90] & 97.693 & [96.637-98.771] & $51.6 \%$ \\
\hline Dept. \#4 & 186.69 & [185.08-188.30] & 91.349 & [90.344-92.376] & $48.9 \%$ \\
\hline Dept. \#5 & 201.23 & [199.01-203.45] & 108.990 & [107.59-110.43] & $54.2 \%$ \\
\hline Dept. \#6 & 192.68 & [190.84-194.51] & 101.000 & [99.858-102.17] & $52.4 \%$ \\
\hline Dept. \#7 & 180.3 & [178.89-181.70] & 88.161 & [87.307-89.032] & $48.9 \%$ \\
\hline Department & Mean & $95 \% \mathrm{Cl}$ & Within Day Variability (SD) & $95 \% \mathrm{Cl}$ & CV\% \\
\hline Dept. \# 1-6 & 191.17 & [190.43-191.90] & 99.159 & [98.699-99.624] & $51.9 \%$ \\
\hline Dept. \#7 & 180.3 & [178.89-181.70] & 88.161 & [87.307-89.032] & $48.9 \%$ \\
\hline
\end{tabular}

Table 1: Variability of daily changes in blood glucose levels measured by the standard deviation of the daily changes and coefficient of variation (CV\%).

\section{Discussion}

The current study documents the outcomes of electronic insulin orders of a basal-bolus regimen that was implemented in one internal medicine department for patients who had an average blood glucose level greater than $200 \mathrm{mg} / \mathrm{dl}$. The outcomes of the treatment were compared to those of patients who were admitted to 6 different internal medicine departments where standardized electronic insulin orders of basal-bolus regimen were not used. In the department in which the standardized electronic insulin orders by CPOE system were utilized, mean blood glucose levels were significantly lower, episodes of hypoglycemia were fewer, and daily variability in blood glucose measurements was less than in the departments that did not use the standardized electronic insulin orders.

Hyperglycemia in hospitalized patients is a common, serious problem, which requires a large portion of health care budgets. Effective management of hyperglycemia is known to prevent complications, as well as to decrease length of hospitalization and mortality rates; both in patients with and without a known history of diabetes. Diabetes management in hospitals is aimed not only to achieve blood glucose control, but also to prevent hypoglycemic events. Consistency in the management of hyperglycemia and the utilization of a physiological basal-bolus insulin treatment regimen promote attainment of these goals [10]. Implementation of protocols for hyperglycemia management for hospitalized patients, as well as self-management in the community, requires simplicity, safety and effectiveness.

A comparative study by Wilson et al. revealed extensive variability in target ranges of glucose blood levels, among 12 published protocols for hyperglycemia management. There were also differences in bolus dosing and in the adjustments of insulin doses according to glucose blood levels. The authors concluded that the ideal insulin infusion protocol should achieve glycemic control within a short time, yield minimal events of hypoglycemia, and have a low risk of error and negligible requirements of medicinal and nursing manpower [11].

In a study that was performed by Farquhar at the Kelowna General Hospital, Kelowna, BC, Canada, a hyperglycemia protocol, based on the Canadian Diabetes Association guidelines, was used for several years in the intensive care unit and in the renal unit. The medical and nursing staff reported that the protocol was very effective and safe, resulting in improved in-hospital management of DM patients and prevention of hypoglycemia episodes [12]. The NICE-SUGAR Study was a large, international trial in which 6104 patients admitted to intensive care units were randomized to undergo intensive control or conventional control of blood glucose levels. Intensive glucose control was found to increase mortality; a blood glucose target of $180 \mathrm{mg} / \mathrm{dL}$ or less resulted in lower mortality than did a target of 81 to $108 \mathrm{mg} / \mathrm{dl}$ [13]. According to the RABBIT 2 Trial, the basal-bolus insulin regimen was found to be preferable to a sliding scale of regular insulin for the management of non-critically ill, hospitalized patients with T2DM [14]. In addition, intensive insulin therapy was found to be beneficial in reducing the mean glucose concentration, but not in preventing its variability. Egi et al. hypothesized that, since variations in glucose concentration might contribute to the pathogenesis mechanisms of hyperglycemia, they may predict mortality in critically ill patients [15]. They performed a large retrospective study, in which 168,337 blood glucose measurements were collected from a cohort of 7,049 critically ill patients, and the standard deviation of the blood glucose concentration was used as a tool to measure its variability. The study revealed that blood glucose variability levels were significantly associated with patients' mortality. Thus, decreasing variability of blood glucose is an important aspect of glucose management.

Following the above findings, the American Diabetes Association recommended that hospitals should develop methods to treat inpatient glucose blood levels [9]. In contrast to previous recommendations for tight glycemic control, a more moderate target range of 140 to 180 $\mathrm{mg} / \mathrm{dl}$ was recommended. To prevent hypoglycemia, glucose targets less than $110 \mathrm{mg} / \mathrm{dl}$ were not recommended. Thus, a clear protocol should be developed that facilitates the induction of corrective changes according to a patient's glucose levels, the rate of changes and individual response to insulin.

Other attempts to develop computerized insulin application protocols have been reported. Crockett et al. studied the safety and efficacy of replacing a paper-based protocol with a computer-guided glucose management system for treating postoperative hyperglycemia in 1,648 patients at a cardiovascular intensive care unit. A significant reduction in hypoglycemia was observed in the computer-guided glucose management system, compared to the paper protocol. Nevertheless, no statistically significant difference in length of stay or mortality was observed between the groups [16]. In another study, Olinghouse examined the implementation of the computerized insulin calculator application connected to HealthConnect [17]. The protocol in that setting was developed in order to reduce nurses' workload. Indeed, the computerized protocol induced a $75 \%$ reduction in nurses' workload burden, and $97 \%$ nursing satisfaction. Magee described a comparative study between a paper insulin protocol and a computerized glucose management system [18]. The computerized glucose management system achieved target glucose blood levels in more than $75 \%$ of all readings, while minimizing the risk of hypoglycemia. A number of studies have demonstrated that the use of computerized glucose control programs are more effective, safer and more reliable than paper programs; and yield high rates of satisfaction of medical staff [19-24].

The patients included in the current analysis represent a range of 
Citation: Dicker D, Marcoviciu D, Beylin V, Belnik Y, Solun B (2015) The Efficacy of Standardized Electronic Insulin Orders In Controlling Blood Glucose Levels In Internal Medicine Departments. J Diabetes Metab 6: 538. doi:10.4172/2155-6156.1000538

Page 5 of 5

clinical conditions that are managed in internal medicine departments. Though data are not available to compare the demographic and clinical characteristics of the patients in the particular departments, we expect that they are similar, since allocation of patients to departments was arbitrary. The present study and others show that computerized insulin orders used to control glucose blood levels prevent unnecessary variation in clinical care and improve healthcare quality, efficiency and safety. Thus, relevant technical and regulatory issues, as well as standardization of protocol development, validation and maintenance should be addressed; after which, electronic hyperglycemia management protocols should be distributed and incorporated to improve patient care during hospitalization.

\section{References}

1. Corriere M Rooparinesingh N, Kalyani RR (2013) Epidemiology of diabetes and diabetes complications in the elderly: an emerging public health burden. Curr Diab Rep 13: 805-813.

2. American Diabetes Association1 (2013) Economic costs of diabetes in the U.S. in 2012. Diabetes Care 36: 1033-1046.

3. Reider J Donihi A, Korytkowski MT (2009) Practical implications of the revised guidelines for inpatient glycemic control. Pol Arch Med Wewn 119: 801-809.

4. Smiley D Umpierrez GE (2010) Management of hyperglycemia in hospitalized patients. Ann N Y Acad Sci 1212: 1-11.

5. Falciglia M Freyberg RW, Almenoff PL, D'Alessio DA, Render ML (2009) Hyperglycemia-related mortality in critically ill patients varies with admission diagnosis. Crit Care Med 37: 3001-3009.

6. McAlister FA Man J, Bistritz L, Amad H, Tandon P (2003) Diabetes and coronary artery bypass surgery: an examination of perioperative glycemic control and outcomes. Diabetes Care 26: 1518-1524.

7. Krinsley JS1 (2003) Association between hyperglycemia and increased hospita mortality in a heterogeneous population of critically ill patients. Mayo Clin Proc 78: 1471-1478.

8. Kosiborod M Inzucchi SE, Krumholz HM, Xiao L, Jones PG, et al. (2008) Glucometrics in patients hospitalized with acute myocardial infarction: defining the optimal outcomes-based measure of risk. Circulation 117: 1018-1027.

9. American Diabetes Association (2015) (13) Diabetes care in the hospital, nursing home, and skilled nursing facility. Diabetes Care 38 Suppl: S80-85.

10. Campbell KB, Braithwaite SS (2004) Hospital management of hyperglycemia. Clinical Diabetes 22: 81-88.
11. Wilson M Weinreb J, Hoo GW (2007) Intensive insulin therapy in critical care: a review of 12 protocols. Diabetes Care 30: 1005-1011.

12. Farquhar A (2007) Practice tips. Insulin protocols for hospital management of diabetes. Can Fam Physician 53: 1668-1669.

13. NICE-SUGAR Study Investigators, Finfer S, Chittock DR, Su SY, Blair D, et al. (2009) Intensive versus conventional glucose control in critically ill patients. N Engl J Med 360: 1283-1297.

14. Umpierrez GE Smiley D, Zisman A, Prieto LM, Palacio A, et al. (2007) Randomized study of basal-bolus insulin therapy in the inpatient management of patients with type 2 diabetes (RABBIT 2 trial). Diabetes Care 30: 2181-2186.

15. Egi M Bellomo R, Stachowski E, French CJ, Hart G (2006) Variability of blood glucose concentration and short-term mortality in critically ill patients. Anesthesiology 105: 244-252.

16. Crockett SE, Suarez-Cavelier J, Accola KD, Hadas LA, Harnage DL, et al. (2012) Risk of postoperative hypoglycemia in cardiovascular surgical patients receiving computer-based versus paper-based insulin therapy. Endocr Pract 18: $529-537$

17. Olinghouse C (2012) Development of a computerized intravenous insulin application (AutoCal) at Kaiser Permanente Northwest, integrated into Kaiser Permanente HealthConnect: impact on safety and nursing workload. Perm $\mathrm{J}$ 16: 67-70.

18. Magee MC1 (2012) Improving IV insulin administration in a community hospital. $J$ Vis Exp : e3705

19. Lee J Fortlage D, Box K, Sakarafus L, Bhavsar D, et al. (2012) Computerized insulin infusion programs are safe and effective in the burn intensive care unit. J Burn Care Res 33: e114-119.

20. Wilinska ME Blaha J, Chassin LJ, Cordingley JJ, Dormand NC, et al. (2011) Evaluating glycemic control algorithms by computer simulations. Diabetes Technol Ther 13: 713-722.

21. Schnipper JL Liang CL, Ndumele CD, Pendergrass ML (2010) Effects of a computerized order set on the inpatient management of hyperglycemia: a cluster-randomized controlled trial. Endocr Pract 16: 209-218.

22. Buchert R, Santer R, Brenner W, Apostolova I, Mester J, et al. (2009) Computer simulations suggest that acute correction of hyperglycaemia with an insulin bolus protocol might be useful in brain FDG PET. Nuklearmedizin 48: 44-54.

23. Dortch MJ, Mowery NT, Ozdas A, Dossett L, Cao H, et al. (2008) A computerized insulin infusion titration protocol improves glucose control with less hypoglycemia compared to a manual titration protocol in a trauma intensive care unit. J Parenter Enteral Nutr 32: 18-27.

24. Boord JB Sharifi M, Greevy RA, Griffin MR, Lee VK, et al. (2007) Computerbased insulin infusion protocol improves glycemia control over manual protocol. J Am Med Inform Assoc 14: 278-287. 